\title{
Management of major bleeding in patients treated with direct oral anticoagulants: from experience to standardized protocols
}

Sara Mascia, ${ }^{1}$ Anna Maria Ferrari, ${ }^{2}$ Nicola Macarone Palmieri, ${ }^{3}$ Elisa Romagnoli, ${ }^{4}$ Chiara Catena, ${ }^{5}$ Serena Scarabottini, ${ }^{5}$ Francesca Mori, ${ }^{5}$ Lucio Brugioni ${ }^{4}$

${ }^{1}$ Emergency Medicine specialist; ${ }^{2}$ Emergency Department, AUSL of Reggio Emilia; ${ }^{3}$ Emergency Room, Arcispedale Santa Maria Nuova, Reggio Emilia; ${ }^{4}$ Internal Medicine and Critical Area Unit, Policlinico di Modena; ${ }^{5}$ Emergency Medicine, University of Modena and Reggio Emilia, Italy

\begin{abstract}
Evaluation of clinical-laboratory-therapeutic management and related clinical outcomes (thrombotic-hemorrhagic complications) of patients undergoing treatment with direct oral anticoagulants (DOACs) during major bleeding. This is a two-year observational retrospective study. 27 cases of major bleeding in patients undergoing a therapy with DOACs presented to the Emergency Department of Arcispedale Santa Maria Nuova (Reggio Emilia Hospital). 16 cases (59\%) underwent reversal of anticoagulation treatment: 19\% using specific reversal therapy (idarucizumab) and $81 \%$ using non-specific agents [4-factor prothrombin complex concentrate (4F-PCC)]. Routine laboratory data were available for all the cases, but only for some patients it was possible to obtain the plasma dosage of the oral anticoagulant. Laboratory data confirm rapid correction of activated partial thromboplastin time within one hour from the reversal of anticoagulation with idarucizumab. The absence of correlation between standard blood tests and plasma drug dosage in patients treated with factor Xa Inhibitors was confirmed too. The management of major bleeding during treatment with DOACs using reversal therapy (idarucizumab) and non-specific reversal agent (4F-PCC) showed minimal thrombotic $(0.3 \%)$ and hemorrhagic $(0.3 \%)$ complications at 90 days; no events occurred after 6 months.
\end{abstract}

\section{Introduction}

Warfarin has been the only drug for the prevention and the treatment of diseases associated with throm-

Correspondence: Lucio Brugioni, Internal Medicine and Critical Area Unit, Policlinico di Modena, via del pozzo 71, 41121 Modena, Italy.

Tel.: +39.059.4225491. E-mail: luciob2362@gmail.com

Key words: New oral anticoagulants; major bleeding; idarucizumab; factor Xa inhibitors; 4-factor prothrombin complex concentrate.

Conflict of interests: the authors declare that they have not received any sponsorship or funding from the pharmaceutical companies of the drugs mentioned in the paper.

Received for publication: 20 October 2019

Revision received: 4 January 2020.

Accepted for publication: 7 January 2020.

This work is licensed under a Creative Commons Attribution NonCommercial 4.0 License (CC BY-NC 4.0).

${ }^{\circ}$ Copyright: the Author(s), 2020

Licensee PAGEPress, Italy

Italian Journal of Medicine 2020; 14:43-48

doi:10.4081/itjm.2020.1224 boembolic risk for almost half a century. Multiple limits of this therapy led to the development of direct oral anticoagulants (DOACs), approved thanks to wide clinical randomized trials showing clearly the non-inferiority of DOACs in terms of reduction of both thromboembolic risk and major bleedings. ${ }^{1,2}$ The first published randomized controlled trials (RCTs) compared DOACs with vitamin $\mathrm{K}$ antagonists in patients with non-valvular atrial fibrillation - ARISTOTLE ${ }^{3}$ for apixaban; RE-LY trial ${ }^{4}$ for dabigatran; ROCKET-AF ${ }^{5}$ for rivaroxaban and ENGAGE AF-TIMI $48^{6}$ for edoxaban -, then RCTs were published about venous thromboembolism.

Main concerns in prescribing DOACs are related to the management of active major bleeding in the Emergency Department (ED) and to still persistent difficulties in laboratory monitoring in urgency settings. ${ }^{7,8}$

To monitor anticoagulant therapy with DOACs, traditional laboratory tests (PT and aPTT) present numerous limits and scarce accuracy; providing only qualitative evaluations of the concentration of the drug and quantitative tests for DOACs plasma level [diluted thrombin time (dTT) and ecarin clotting assay for dabigatran and measurement of anti-FXa activity for rivaroxaban, apixaban and edoxaban] are available only in some laboratory.

In case of major bleeding, in our reality, the ther- 
apeutic choice for reversal of anticoagulant activity are specific antidotes (at the time of the study they were available only for dabigatran) or the 4-factor prothrombin complex concentrate (4F-PCC) for other DOACs. There are different formulations of prothrombin concentrates: PCC with 3 factors (II, IX, X; UMAN COMPLEX ${ }^{\circledR} /$ PROTROMPLEX $\left.{ }^{\circledR}\right)$, with 4 factors containing factor VII in addition to protein $\mathrm{S}$ and $\mathrm{C}$ (CONFIDEX ${ }^{\circledR}$, PRONATIV $\left.{ }^{\circledR}\right)$, the recombinant active factor VII (rFVIIa) and the activated prothrombin complex with 4 factors (aPCC, FEIBA ${ }^{\circledR)}$.

Effectiveness of clotting factors concentrates has been described in patients undergoing therapy with AVK with major bleeding. ${ }^{9-11}$

However, their effectiveness in bleeding associated with DOACs has not been proven by large randomized controlled studies. ${ }^{12-16}$ Anyway, the use of $4 \mathrm{~F}-\mathrm{PCC}$ is the therapeutic nonspecific strategy more widely used to revert the activity of the direct inhibitors of $\mathrm{FXa}^{12,18-21}$ while there is even less certainty about the aPCC usage..$^{22,23}$

The best strategy remains the use of specific antidotes, allowing an optimal management of those patients who present to the ED with major bleeding or requiring emergency surgery or procedure. Idarucizumab has been approved and it is already in use for reversal dabigatran activity, while the antidote for direct inhibitors of FXa (andexanet alpha) has been approved by FDA and EMA has granted a conditional authorization.

The studies that analyzed the efficacy and safety of these antidotes are: REVERSE AD (dabigatranidarucizumab), ANNEXA-A and ANNEXA-R (andexanet-inhibitor FXa).

The aim of our study is to observe retrospectively how in our reality (Emergency Department of Arcispedale Santa Maria Nuova, Reggio Emilia) major bleedings in course of DOACs were managed. We collected data about the chosen strategy of reversal, coagulation assay, plasma levels used and clinical outcome of patients.

\section{Materials and Methods}

In this retrospective single-center study we enrolled a cohort of 27 consecutive patients who presented in a two-year period (March 2016-March 2018) at the ED of Santa Maria Nuova Hospital in Reggio Emilia with a major hemorrhage (defined according to ISTH classification ${ }^{24}$ ) during treatment with direct oral anticoagulants.

Numerous data were collected for each patient and inserted into a database, including the following: i) previous ischemic and/or hemorrhagic events; ii) type of DOACs, dosage and time elapsed since the last in- take, indication for anticoagulant treatment: atrial fibrillation (AF) associated or not with previous stroke, recent or previous diagnosis of venous thromboembolism (VTE) and duration of treatment (if undertaken during the previous 3 months); iii) data about the admission to the emergency room: color code assigned in triage; hemodynamic and/or neurological state of the patient on arrival; iv) plasma level of the drug, when available, and coagulation assay at $0-1-12 \mathrm{~h}$ (PT and aPTT for dabigatran; PT for rivaroxaban, apixaban and edoxaban); v) dosage and type of drug chosen for the reversal of anticoagulation activity and the time elapsed between the access to ED and its administration; vi) clinical outcomes: intrahospital death, ischemic and/or hemorrhagic events during follow-up (at 30-90 days and at 6 months detected by computerized patient archive).

All the data were used for subsequent simple statistical analysis.

\section{Results}

Of the 27 patients enrolled, 4 were treated with dabigatran, 10 with rivaroxaban, 12 with apixaban and only one with edoxaban. Among them, 19 were on anticoagulant therapy in primary prevention for nonvalvular atrial fibrillation, while 3 were in secondary prevention for AF. The remaining 5 patients in the study were on anticoagulant treatment following VTE.

In the study group, 4 patients (15\%) had started anticoagulant therapy less than 3 months before the major hemorrhagic event.

In ED 52\% $(n=14)$ of patients showed gastro-intestinal bleeding and $48 \%(n=13)$ intracranial bleeding (Table 1). Among the latter, in 6 cases bleeding was due to post-traumatic events ( 1 case of subarachnoid hemorrhage and 5 subdural hemorrhages); the other 7 patients developed spontaneous events (4 deep-thalamus localization hemorrhages/base nuclei and 3 lobe hemorrhages).

Table 2 shows data that we have collected.

Table 1. Number and site of major bleeding.

\begin{tabular}{lccc}
\hline Type of drug & Number of & \multicolumn{2}{c}{ Bleeding site } \\
& major bleeding & ICH & GI \\
\hline Dabigatran & 4 & 3 & 1 \\
\hline Rivaroxaban & 10 & 2 & 8 \\
\hline Apixaban & 12 & 8 & 4 \\
\hline Edoxaban & 1 & 1 & \\
\hline Total & 27 & 13 & 14 \\
\hline
\end{tabular}

$\mathrm{ICH}$, intracerebral hemorrhage; GI, gastrointestinal. 


\section{Patients treated with reversal of anticoagulation activity}

Sixteen patients $(59 \%)$ were treated with reversal of anticoagulant activity.

For three patients receiving dabigatran, idarucizumab $5 \mathrm{~g}$ was administered intravenously as twobolus doses of $2.5 \mathrm{~g}$ no more than $15 \mathrm{~min}$ apart. Two cases had intracranial bleeding and one had digestive bleeding. All patients had taken the last dose less than $7 \mathrm{~h}$ before the hemorrhagic event. Only for one patient DOACs plasma concentration was performed at time 0 and at 12-h intervals for control (which confirmed dTT normalization). In all 3 cases, routine laboratory tests showed normal renal function; aPTT at time 0 was prolonged with subsequent normalization in all cases within one hour after administration of the specific antidote. The mean time between presentation to the ED and administration of idarucizumab was $85 \mathrm{~min}$.

Five patients undergoing therapy with rivaroxaban were all treated with a 4F-PCC. Two of them had intracranial bleeding and three of them had major gastrointestinal bleeding. Both patients with cerebral hemorrhage had taken the last dose between 13 and 16 hours earlier: the dosage of $25 \mathrm{IU} / \mathrm{kg}$ 4F-PCC was chosen. In blood tests renal function was normal, plasma FXa activity showed different plasma concentrations (for one patient $20 \mathrm{ng} / \mathrm{mL}$ and for another $>200 \mathrm{ng} / \mathrm{mL}$ ) compared to a qualitative coagulation test (PT) that was normal in both cases. The remaining three patients with a gastro-intestinal hemorrhage had taken the last dose of the drug within the previous 3 hours. They were all treated with doses of 4F-PCC ranging between 25 and $37.5 \mathrm{IU} / \mathrm{kg}$. Laboratory tests showed conflicting pictures: in two patients PT was prolonged and for one of them the anti-FXa activity was $>300 \mathrm{ng} / \mathrm{mL}$; in the third patient, PT was normal and the plasma concentration of the drug was $20 \mathrm{ng} / \mathrm{mL}$.

In the eight patients treated with apixaban therapy, the anticoagulation activity was reverted with 4F-PCC (Pronativ ${ }^{\circledR}$ ) using doses between 25 and $50 \mathrm{IU} / \mathrm{kg}$ : all of them presented cerebral bleeding (post traumatic in three cases). Five cases had taken the last dose of the drug less than $5 \mathrm{~h}$ earlier, the remaining 3 between 13 and $17 \mathrm{~h}$ earlier. All had normal renal function. In pa- tients who had taken the drug between 13 and $17 \mathrm{~h}$ before the hemorrhage, laboratory tests showed plasma concentrations of apixaban between 80 and $180 \mathrm{ng} / \mathrm{mL}$ with a normal PT in $100 \%$ of patients; in patients who had taken the dose earlier, the plasma dose of the drug was $>200 \mathrm{ng} / \mathrm{mL}$. The average time between ED access and reversal therapy was 64 minutes.

All patients with gastrointestinal bleeding received endoscopic treatment beyond $24 \mathrm{~h}$ after arrival at ED.

\section{Patients not treated with reversal of anticoagulation activity}

Eleven patients have not undergone reversal therapy: 10 had gastrointestinal hemorrhage (all treated with FXa inhibitors) and 1 had cerebral hemorrhage.

The case of intracranial bleeding was the only one among patients on dabigatran treatment not to be managed by administration of the specific antidote. This choice derived from the finding of a minimal blood quota in the tomographic scan (two spontaneous intracranial hemorrhagic petechiae). The last intake of the drug was $14 \mathrm{~h}$ earlier in this patient; the coagulation pattern of the biohumoral tests was altered (aPTT>2), dTT was not performed.

Among the five patients receiving rivaroxaban: 3 patients had eGFR $>60 \mathrm{~mL} / \mathrm{min}$ and two $<35 \mathrm{~mL} / \mathrm{min}$ (calculated with Cockcroft-Gault formula). The drug was taken more than $24 \mathrm{~h}$ earlier and all were hemodynamically stable. Laboratory tests showed discrepancies: in two cases PT was prolonged but in one of them the plasma concentration of the drug was $<30$ $\mathrm{ng} / \mathrm{mL}$; in the other three cases, PT was normal but only for one patient the plasma concentration of the drug was assessed, which was $60 \mathrm{ng} / \mathrm{mL}$. Four of these patients were treated endoscopically within $3 \mathrm{~h}$ from the arrival to the ED.

All patients receiving apixaban had normal renal function and altered blood coagulation with PT prolongation. The plasma drug concentration was only required in two patients and for both patients it was $>160 \mathrm{ng} / \mathrm{mL}$. Two had taken the drug less than $4 \mathrm{~h}$ before and the other two between 11 and 13 hours before. Of them, one patient died within one hour from the arrival in the emergency room for hemor-

Table 2. Types of treatment.

\begin{tabular}{lcc}
\hline Type of drug (number of bleeding) & Specific reversal agents & Non-specific reversal agents \\
\hline Dabigatran (4) & 3 & $/$ \\
\hline Rivaroxaban (10) & $/$ & 5 \\
\hline Apixaban (12) & $/$ & 8 \\
\hline Edoxaban (1) & $/$ & $/$ \\
\hline
\end{tabular}


rhagic shock; the others presented hemodynamically stable.

The only patient on edoxaban did not undergo recoagulation, because clinical stability at the onset and the last dose of DOACs had been taken more than 24 $\mathrm{h}$ earlier.

Only one of the patients with intracranial bleeding required neurosurgery approach: he presented an acute subdural hemorrhage and was treated with clotting factors concentrates (4F-PCC).

\section{Clinical outcomes}

Four deaths (15\% of the total) occurred within 72 hours most likely in relation to the severity of the clinical condition at the onset: they were all hemodynamically unstable, treated with direct factor Xa inhibitors and received reversal therapy with clotting factors concentrates (4F-PCC).

During the follow-up, 19 patients (70\%) resumed anticoagulant therapy, all with direct oral anticoagulants.

Only one case of thrombotic complication (recurrent femoral-popliteal deep vein thrombosis) has been reported at 90 days: this patient was previously treated with apixaban for VTE and received 4F-PCC for a subependymal cerebral hemorrhage, furthermore, after the acute event, the patient did not restart any anticoagulant therapy.

At 90 days only one hemorrhagic complication developed too: one case of minor hemorrhage (rectal bleeding) was observed in a patient treated with rivaroxaban for $\mathrm{AF}$.

Thrombotic or hemorrhagic complications at 6 months have not been registered in any case,

\section{Conclusions}

Data collected reveal the great variability in the management of major bleeding during DOACs in an emergency department.

The effectiveness and safety of treatment with idarucizumab is already known and confirmed in our study.

Generally, routine coagulation test (PT and aPTT) does not provide an accurate assessment of DOACs anticoagulant effect. For dabigatran, aPTT may provide a qualitative assessment of dabigatran activity while aPTT and also PT cannot be used for any meaningful evaluation of FXa inhibitory effect. In particular, they should not play any role in guiding the treatment of major bleeding and the possible administration of reversal. In our study the use of idarucizumab allowed the correction of both dTT at $12 \mathrm{~h}$ and the aPTT within one hour.

The decision to administer reversal agents was mainly guided by the type of hemorrhage, patient clinical condition and time from last drug intake (for drugs with well-known elimination half-life): all intracranial hemorrhages, being considered major hemorrhages, have been treated, while some cases of gastrointestinal hemorrhage have been treated without reversal because causal and also operative therapy could be applied.

In the emergency setting of a major bleeding, it would be desirable to perform the quantification of DOACs plasma levels but these tests are not yet widely available, not low-cost and reference ranges for peak and through levels are not standardized, although complex technology (most routine coagulometers are capable of measuring DOACs plasma levels within $<30 \mathrm{~min}$ ) is not required. Management protocols shared with laboratory are necessary in order to implement the use of such data that could really guide the management of the patient with major bleeding during DOACs. In particular, if plasma level has not been confirmed, the administration of 4F-PCCs and specific antidotes (drugs with high-cost and with thrombotic high-risk) is not indicated.

In our study, the timing of endoscopic examination in patients with gastrointestinal hemorrhage was very variable (within $3 \mathrm{~h}$ or more than $24 \mathrm{~h}$ ): the possibility for DOACs to predict the expected time of normalization of plasma levels based on renal function allows to safely delay the endoscopic examination; the correct timing shall be always agreed with the reference specialist, according to the patient clinical status.

Thrombotic and/or hemorrhagic events were minimal at 90 days $(0.3 \%)$ and these were absent at 6 months, underlying the effectiveness of the treatment of major bleeding if it is performed according to available scientific evidence. In addition, these data demonstrate the need for short-term evaluation for the timing of anticoagulation reinitiation.

Therefore, in all emergency departments it is necessary to create and to follow standardized multidisciplinary protocols based on scientific evidence available for the management of major bleeding during treatment with DOACs.

Although this is a small observational retrospective study carried out on a limited number of patients, our work emphasizes the great variability in the management of such emergency and the absolute necessity to follow shared standardized protocols. In this regard, in case of a major hemorrhage in a patient on DOACs treatment, our study further supports the adoption of the practical indications reported by HERA Guidelines $2018^{25}$ and ACC consensus $2017,{ }^{26}$ which first require a classification of hemorrhagic events to consequently apply the specific treatment (Figure 1; Tables 3 and 4). 


\section{Assessing bleed severity}

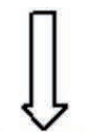

minor bleeding: (epistaxis, conjunctival hemorrhage, ecchymosis, hematuria without anemia): in this case no therapeutic measures are required.

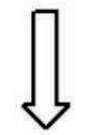

non-life-threatening major bleeding: causal therapy to stop the bleeding and treatment interruption.<smiles>[C]=C</smiles>

life threatening bleeding (intracranial hemorrhage, intraocular, intraspinal, thoracic, intra-abdominal, retroperitoneal, intrarticular, intramuscular bleeds): benefit from DOACs reversal in addition to the standard measures

Figure 1. Classification of hemorrhagic events.

Table 3. Management of non-life-threatening major bleeding.

Direct thrombin inhibitors (dabigatran)

FXa inhibitors (apixaban, rivaroxaban, edoxaban)

- Identify the drug, dosing regimen and inquire about last intake

- Urgently perform the specific laboratory tests for assessing the anticoagulant activity (diluted thrombin time and ecarin clotting assay for dabigatran; chromogenic anti-Xa assay for FXa inhibitors)

- Stop DOACs

- Consider the use of tranexamic acid ( 1 gr i.v. repeated every $6 \mathrm{~h}$ if needed) especially in bleeding following trauma

- Consider activated charcoal for known recent ingestion $(<2 \mathrm{~h}$, only for dabigatran use)

- Fluid replacement and maintain diuresis

- Execute, when possible, supportive measures for mechanical hemostasis (endoscopic, surgical, interventional radiology, etc.)

- Estimate normalization of plasma levels:

- Normalization of plasma levels in 12-24 h

i) normal renal function $12-24 \mathrm{~h}$;

ii) $\mathrm{CrCl}</ \min : 36-48 \mathrm{~h}$

- Consider idarucizumab (see below)

Table 4. Management of life-threatening bleeding.

Direct thrombin inhibitors (dabigatran)

FXa inhibitors (apixaban, rivaroxaban, edoxaban)

- All of the above

- All of the above

- Direct reversal with idarucizumab 5 gr i.v. in two boluses

- 4F-PCC $25-50 \mathrm{UI} / \mathrm{kg}$ of $2.5 \mathrm{gr}$ no more than $15 \mathrm{~min}$ apart

- Activated PCC or human recombinant coagulation factor VII can be considered only if 4F-PCC is unavailable

- Fresh frozen plasma not as reversal agent 


\section{References}

1. Larsen TB, Rasmussen LH, Skjoth F, et al. Efficacy and safety of dabigatran etexilate and warfarin in "real-world" patients with atrial fibrillation: a prospective nationwide cohort study. J Am Coll Cardiol 2013;61:2264-73.

2. Lauffenburger JC, Farley JF, Gehi AK, et al. Effectiveness and safety of dabigatran and warfarin in real-world US patients with non-valvular atrial fibrillation: a retrospective cohort study. J Am Heart Assoc 2015;4:e001798.

3. Granger CB, Alexander JH, McMurray JJ, et al.; ARISTOTLE Committees and Investigators. Apixaban versus warfarin in patients with atrial fibrillation. N Engl J Med 2011;365:981-92.

4. Connolly SJ, Ezekowitz MD, Yusuf S, et al.; RE-LY Steering Committee and Investigators. Dabigatran versus warfarin in patients with atrial fibrillation. N Engl J Med 2009;361:1139-51.

5. Patel MR, Mahaffey KW, Garg J, et al.; ROCKET AF Investigators. Rivaroxaban versus warfarin in nonvalvular atrial fibrillation. N Engl J Med 2011;365:883-91.

6. Giugliano RP, Ruff CT, Braunwald E, et al.; ENGAGE AF-TIMI 48 Investigators. Edoxaban versus warfarin in patients with atrial fibrillation. $\mathrm{N}$ Engl J Med 2013;369:2093-104.

7. Pollack CV Jr. Coagulation assessment with the new generation of oral anticoagulants. Emerg Med J 2015 [Epub ahead of print].

8. Kakkos SK, Kirkilesis GI, Tsolakis IA. Editor's choice efficacy and safety of the new oral anticoagulants dabigatran, rivaroxaban, apixaban, and edoxaban in the treatment and secondary prevention of venous thromboembolism: a systematic review and meta-analysis of phase III trials. Eur J Vasc Endovasc Surg. 2014;48:565-75.

9. Voils SA, Baird B. Systematic review: 3-factor vs 4-factor prothrombin complex concentrate for warfarin reversal: does it matter? Thromb Res 2012;130:833-40.

10. Voils SA, Holder MC, Premraj S, et al. Comparative effectiveness of 3- vs 4-factor prothrombin complex concentrate for emergent warfarin reversal. Thromb Res 2015; 13:595-8.

11. Mangram A, Oguntodu OF, Dzandu JK, et al. Is there a difference in efficacy, safety, and cost-effectiveness between 3-factor and 4-factor prothrombin complex concentrates among trauma patients on oral anticoagulants? J Crit Care 2016;33:252-6.

12. Gavva C, Reddy M, Sarode R. Four-factor prothrombin complex concentrates: effectiveness in the reversal of anticoagulation. Int J Clin Transf Med 2017 [Epub ahead of print].

13. Lindahl TL, Wallstedt M, Gustafsson KM, et al. More efficient reversal of dabigatran inhibition of coagulation by activated prothrombin complex concentrate or recombinant factor VIIa than by four-factor prothrombin complex concentrate. Thromb Res 2015;135:544-7.
14. Grottke O, Aisenberg J, Bernstein R, et al. Efficacy of prothrombin complex concentrates for the emergency reversal of dabigatran-induced anticoagulation. Crit Care 2016;20:115.

15. Díaz MQ, Borobia AM, Núñez MA, et al. Use of prothrombin complex concentrates for urgent reversal of dabigatran in the Emergency Department. Haematologica 2013;98:e143-4.

16. Barco S, Whitney Cheung Y, Coppens M, et al. In vivo reversal of the anticoagulant effect of rivaroxaban with four-factor prothrombin complex concentrate. $\mathrm{Br} \mathrm{J}$ Haematol 2016;172:255-61.

17. Zahir H, Brown KS, Vandell AG, et al. Edoxaban effects on bleeding following punch biopsy and reversal by a 4-factor prothrombin complex concentrate. Circulation 2015;131:82-90.

18. Eerenberg ES, Kamphuisen PW, Sijpkens MK, et al. Reversal of rivaroxaban and dabigatran by prothrombin complex concentrate: a randomized, placebo-controlled, crossover study in healthy subjects. Circulation 2011;124:1573-9.

19. Levi M, Moore KT, Castillejos CF, et al. Comparison of three-factor and four-factor prothrombin complex concentrates regarding reversal of the anticoagulant effects of rivaroxaban in healthy volunteers. J Thromb Haemost 2014; 12:1428-36.

20. Albaladejo P, Samama C-M, Sie' P, et al; GIHP-NACO Study Group. Management of severe bleeding in patients treated with direct oral anticoagulants: an observational registry analysis. Anesthesiology 2017;127:111-20.

21. Majeed A, Agren A, Holmstrom M, et al. Management of rivaroxaban- or apixaban-associated major bleeding with prothrombin complex concentrates: a cohort study. Blood 2017;130:1706-12.

22. Baxter Healthcare Corporation. FEIBA NF (anti-inhibitor coagulant complex), nanofiltered and vapor heated. Available from: http://www.feiba.com/us/forms/feiba_nf_pi. pdf Accessed: February 12, 2017.

23. Dibu JR, Weimer JM, Ahrens C, et al. The role of FEIBA in reversing novel oral anticoagulants in intracerebral hemorrhage. Neurocrit Care 2016;24:413-9.

24. Schulman S, Angeras U, Bergqvist D, et al. Definition of major bleeding in clinical investigations of antihemostatic medicinal products in surgical patients. J Thromb Haemost 2010;8:202-4.

25. Steffel J, Verhamme P, Potpara TS, et al; ESC Scientific Document Group. The 2018 European Heart Rhythm Association Practical Guide on the use of non-vitamin Kantagonist oral anticoagulants in patients with atrial fibrillation. Europace 2018;20:1231-42.

26. Tomaselli GF, Mahaffey KW, Cuker A, et al.; 2017 ACC Expert Consensus Decision Pathway on Management of Bleeding in Patients on Oral Anticoagulants. A Report of the American College of Cardiology Task Force on Expert Consensus Decision Pathways. J Am Coll Cardiol 2017 [Epub ahead of print]. 